\title{
A Multilayer Feed Forward Small-World Neural Network Controller and Its Application on Electrohydraulic Actuation System
}

\author{
Xiaohu Li, Feng Xu, Jinhua Zhang, and Sunan Wang \\ School of Mechanical Engineering, Xian Jiaotong University, Xian 710049, China \\ Correspondence should be addressed to Xiaohu Li; li.xiaohu@mail.xjtu.edu.cn
}

Received 26 March 2013; Accepted 21 May 2013

Academic Editor: Tao Zou

Copyright (C) 2013 Xiaohu Li et al. This is an open access article distributed under the Creative Commons Attribution License, which permits unrestricted use, distribution, and reproduction in any medium, provided the original work is properly cited.

\begin{abstract}
Being difficult to attain the precise mathematical models, traditional control methods such as proportional integral (PI) and proportional integral differentiation (PID) cannot meet the demands for real time and robustness when applied in some nonlinear systems. The neural network controller is a good replacement to overcome these shortcomings. However, the performance of neural network controller is directly determined by neural network model. In this paper, a new neural network model is constructed with a structure topology between the regular and random connection modes based on complex network, which simulates the brain neural network as far as possible, to design a better neural network controller. Then, a new controller is designed under smallworld neural network model and is investigated in both linear and nonlinear systems control. The simulation results show that the new controller basing on small-world network model can improve the control precision by $30 \%$ in the case of system with random disturbance. Besides the good performance of the new controller in tracking square wave signals, which is demonstrated by the experiment results of direct drive electro-hydraulic actuation position control system, it works well on anti-interference performance.
\end{abstract}

\section{Introduction}

As we know, neural network is an adaptive function estimator needless to know the determined math relationship between input and output, and it also has good adaptability and learning ability. These features make it very suitable to be used as intelligent controller for complex systems. Many researches show that the neural network controller is especially suitable for those uncertain or nonlinear control objects, which make it have a wide application prospect in the field of intelligent control. Obviously, the constructed neural network model directly determines the quality of the neural network controller; in short, the architecture design is critical for neural network model. At present, the structures of artificial neural network model are often designed as feedback, feed forward, single neuron, multilayer, and so on. As such, it is an important problem worthy of discussion whether these structures of neural network models are optimal and if they are able to reflect the real human brain neural network structure or not.

Recent researches have shown that the structure topology and function of human brain neural network are closely related to each other; the connection mode of brain neural network structure topology offers the possibility for different brain areas to mutual collaboration [1-6]. This collaboration is mainly due to the large number of neurons in human brain, which means that the simple structure topology and function of a single neuron are multiplied by the large number of neurons. All those neurons connecting with each other by nerve fibers according to a certain kind of connection mode can make up highly complex human brain neural network. Unfortunately, the existing artificial neural network models are simple simulation of the biological neural network in structure topology and function. The common connection modes of artificial neural network can be divided into feed forward, feedback, single layer, multilayer, and so forth, all 
of which can be regarded as regular structure topology. In the past few decades, lots of researches have been carried out regarding artificial neural network with regular structure topology [7-10]. Although the bioneurological studies have shown that neural network inherently has random features on structure topology, the research of artificial neural network with random structure topology is relatively rarely reported. Consequently, it is essential to design a neural network model with stochastic characteristic structure, which will not only help in obtaining an optimal network performance, but also be a more realistic reflection of the structural features of the brain neural network.

At present, there are two typical methods to reflect the random features of neural network: one is to adopt random connection weight between the neurons of neural network; the other is to use random neuron activation function [11]. But neither of the methods mentioned above can really reveal the random features on neural network structure topology. New achievements of complex network have provided a new method for constructing neural network model with random feature structure. Watts and Strogatz researched on the structure topology of many realistic complex networks and defined the intermediate network between completely regular and random networks as small-world network (referred to as W-S model) [12]. Many studies on complex network have shown that the realistic networks, such as disease transmission network, social network, food chain network, metabolic network, and so forth, are all small-world network on structure topology [13-18]. Meanwhile, scholars discovered that biological neural networks are also smallworld network [19-23]. All of these small-world networks have random characteristics in structure. Obviously, if the structure topology of artificial neural network is built as a small-world one, it will really reflect the structure topology of the biological neural network. Erkaymaz et al. and Simard et al. rewired the links of multilayer feed forward neural network to build a small-world neural network model [24, 25]. However, in the construction process, it is apparent that the unchanging number of rewiring means a lack of randomness on structure topology, which is not consistent with W-S model. Therefore, it is necessary to rebuild a new neural network model, which relies on the rewiring probability. As self-learning is an important characteristic of artificial neural network, control application can be used to assess the performance of multilayer feed forward smallworld neural network model. Since the new controller does not require a precise mathematical model, it can be applied to the electrohydraulic actuation system, with the help of which we may probe the features of small-world neural network model as well as its controller.

In this paper, firstly, the multilayer feed forward smallworld neural network model is built up according to the $\mathrm{W}$ $S$ model. Secondly, the mathematical model of small-world neural network is briefly described. Finally, a new controller with a small-world neural network model is designed for system control, which will not only explore the control performance of small-world neural network, but also compare control precision and anti-interference of small-world neural network with those of regular neural network. The performance of the controller is also verified by experiment on electrohydraulic actuation system.

\section{Model Construction}

In the multilayer feed forward neural network model, the $i$ th neuron of the $l$ th layer $v_{i}^{l}$ only connects with its neighbor neurons, which belong to neuron sets $V^{l-1}$ and $V^{l+1}$. All of these links are feed forward, and there are no links between neurons of the same layer. As the links of each neuron are similar, this network structure topology can be regarded as regularity $[26,27]$. Assuming that the number of neurons in each layer is $n_{l}$, and the number of layers of neural network is $L$ (including the input layer and output layer), the regular network structure topology is shown in Figure 1(a) when rewiring probability $p=0$. The operating mode of regular network is that input signals pass through the hidden layers, and transfer forwardly layer by layer, until they reach the output layer.

Referring to the construction process of W-S model, a multilayer feed forward small-world neural network is built up in the literature [24]. In the construction process, the regular links of multilayer feed forward neural network are reconnected, but as a result of the determinate number of links and the network construction different from W-S model, the rewiring probability $p$ is not used to reconstruct the network. Thus it cannot fully reflect the construction ideology of W-S model. Taking into account that the rewiring probability $p$ has a direct impact on the generated network structure and characteristics, this paper proposes an algorithm to construct the multilayer feed forward small-world neural network model according to the rewiring probability $p$, and the process of algorithm is described as follows.

(1) Generate the same number of neurons in each layer, and connect neighbor neurons with feed forward links. Thus the multilayer feed forward regular neural network model is built, as shown in Figure 1(a), and the connection mode of this model is regular.

(2) With rewiring probability $p$, disconnect the links from neuron $i$ of the $l$ th layer to neuron $j$ of the $(l+1)$ th layer in regular network; then randomly select neuron $j^{\prime}$ ahead the $(l+1)$ th layer to rewire; the new long links are not reconnection or self-loop. Clearly, if the links of the $(L-1)$ th layer disconnect, they could not generate new long links. Therefore, the last two layers cannot be rewired.

(3) Repeat (2), until all the links are rewired besides those of the last two layers.

Figure 1(a) shows that when $p=0$, the connection mode of neural network model is completely regular, in which each neuron maintains the same number of links with adjacent neurons and this neural network model is commonly used. When $p=1$, as shown in Figure 1(c), all original links in the regular neural network model (except for the last two layers) are rewired into links with completely random features under disordered structure topology, forming a completely random 


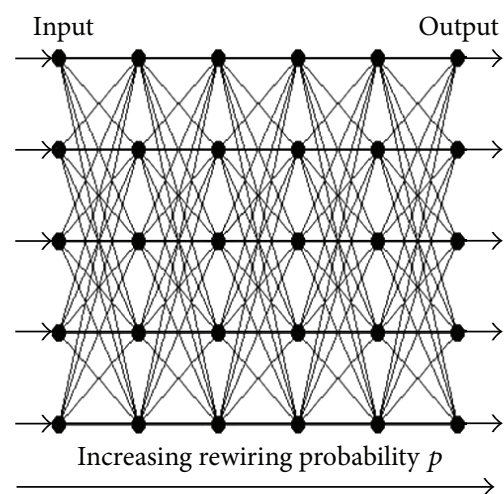

(a) $p=0$

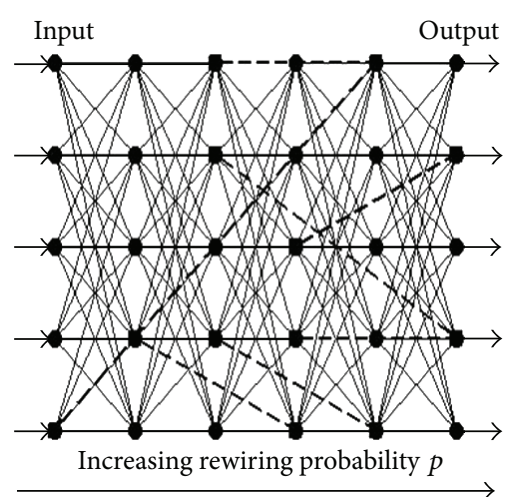

(b) $p=0.05$

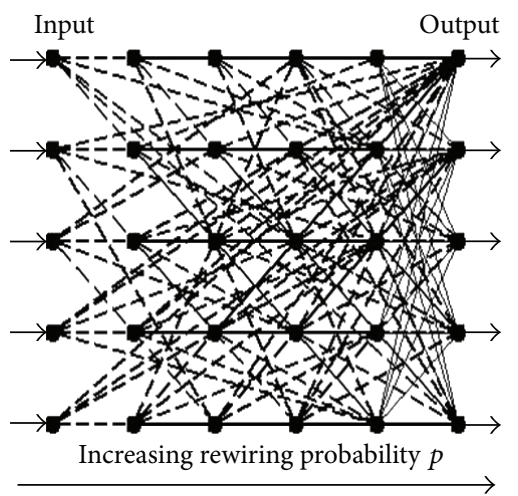

(c) $p=1$

FIGURE 1: Process of construction multilayer feed forward small-world neural network.

neural network model. If $0<p<1$ (e.g., $p=0.05$ ), long cross-layer links will be generated by rewiring probability $p$, and this structure topology is between completely regular and random, as shown in Figure 1(b). Obviously, this connection structure topology is an intermediate form from regular to random, which is in consistent with the ideology of W-S model. Therefore, this model is defined as multilayer feed forward small-world neural network model, and the smaller $p$ is, the fewer there exist the long links in the new network model.

\section{Network Model}

In order to describe multilayer feed forward small-world neural network model distinctly, graph theory is utilized. Each neuron in multilayer feed forward small-world neural network model can be seen as a node in the graph. The links within all neurons are edges of the graph. The node set of small-world neural network model can be defined as

$$
V=\left\{V^{l} \mid l=1,2, \ldots, L\right\},
$$

Where $V^{l}=\left\{v_{i}^{l} \mid i=1,2, \ldots, n_{l}\right\}$ is the node subset of the $l$ th layer, $L$ is the total layer number of the neural network model, $v_{i}^{l}$ is the $i$ th node of the $l$ th layer, and $n_{l}$ is the node number of each layer.

Suppose the connection matrix of the neural network model is

$$
\mathbf{W}=\left\{\mathbf{W}^{1}, \ldots, \mathbf{W}^{1}, \ldots, \mathbf{W}^{L-1}\right\}
$$

where $\mathbf{W}^{l}(l=1,2, \ldots, L-1)$ is the connection submatrix of the $l$ th layer, and $\mathbf{w}_{i}^{l}$ is the connection vector of $V^{l} \rightarrow v_{i}^{l+1}$ :

$$
\begin{gathered}
\mathbf{w}_{i}^{l}=\left(w_{1 i}^{l}, w_{2 i}^{l}, \ldots, w_{n_{l} i}^{l}\right)^{T}, \\
\mathbf{W}^{l}=\left(\mathbf{w}_{1}^{l}, \mathbf{w}_{2}^{l}, \ldots, \mathbf{w}_{n_{l}}^{l}\right),
\end{gathered}
$$

where $i=1,2, \ldots, n_{l}$, and $w_{i j}^{l} \in \mathbb{R}$ is the connection weight of $v_{i}^{l} \rightarrow v_{j}^{l+1}$; if neuron $i$ of the $l$ th layer connects with neuron $j$ of the $(l+1)$ th layer, $w_{i j}^{l} \neq 0$, contrariwise, $w_{i j}^{l}=0$. Therefore, the regular neural network model connection matrix can be expressed as

$$
\mathbf{W}=\begin{gathered}
V^{1} \\
V^{2} \\
V^{2} \\
\vdots \\
V^{L-1} \\
V^{L}
\end{gathered}\left[\begin{array}{cccccc}
0 & \mathbf{W}^{1} & 0 & \cdots & 0 & 0 \\
0 & 0 & \mathbf{W}^{2} & \cdots & 0 & 0 \\
\vdots & \vdots & \vdots & \ddots & \vdots & \vdots \\
0 & 0 & 0 & \cdots & 0 & \mathbf{W}^{L-1} \\
0 & 0 & 0 & \cdots & 0 & 0
\end{array}\right] .
$$

As for the multilayer small-world neural network model, due to the rewiring, the connection matrix even becomes

$$
\begin{aligned}
& \begin{array}{llllll}
V^{1} & V^{2} & \cdots & \cdots & V^{L-1} & V^{L}
\end{array} \\
& \mathbf{W}=\begin{array}{c}
V^{1} \\
V^{2} \\
\vdots \\
V^{L-1} \\
V^{L}
\end{array}\left[\begin{array}{ccccccc}
0 & \mathbf{W}^{1^{\prime}} & B_{1}^{1} & \cdots & B_{1}^{L-3} & B_{1}^{L-2} \\
0 & 0 & \mathbf{W}^{2^{\prime}} & \cdots & B_{2}^{L-4} & B_{2}^{L-3} \\
\vdots & \vdots & \vdots & \ddots & \vdots & \vdots \\
0 & 0 & 0 & \cdots & 0 & \mathbf{W}^{L-1^{\prime}} \\
0 & 0 & 0 & \cdots & 0 & 0
\end{array}\right] .
\end{aligned}
$$

As the reconnections of the last two layers in the neural network model do not exist $\left(\mathbf{W}^{L-1^{\prime}}=\mathbf{W}^{L-1}, \mathbf{W}^{l^{\prime}}=\left(w_{i j}^{l \prime}\right)_{n_{l} \times n_{l}}\right)$ is the reconnection matrix of $\mathbf{W}^{l}$, the elements of this matrix can be deformed into the following equation:

$$
w_{i j}^{l \prime}= \begin{cases}w_{i j}^{l}, & \operatorname{rand}_{i j} \geq p \\ 0, & \operatorname{rand}_{i j}<p,\end{cases}
$$

where $\operatorname{rand}_{i j}$, which is generated at the $i$ th row and the $j$ th column in the matrix, is a random number between 0 and 1. $\mathbf{B}_{l}^{l \prime}=\left(b_{n_{1} n_{2}}^{l l \prime}\right)_{n_{l} \times n_{l}}$ is the reconnection submatrix of $V^{l} \rightarrow V^{l \prime}, l \in\{1,2, \ldots, L-2\}, l^{\prime} \in\{3,4, \ldots, L\}, n_{1}, n_{2} \in$ $\left\{1,2, \ldots, n_{l}\right\}$.

From the connection matrix $\mathbf{W}$, it can be seen that the neurons of the $l$ th layer connect the $(l+1)$ th layer's neurons with the probability $(1-p)$ and connect the neurons of 
the layer following the $(l+1)$ th layer with the probability $p$. If the number of network layers and neurons is large and the rewiring probability $p$ is small, the connection matrix will be a sparse matrix. In short, the neurons of the $l$ th layer connect not only with the neurons of the $(l-1)$ th layer in multilayer feed forward small-world neural network model; they also connect with the neurons of the $1 \sim(l-2)$ th layers in accordance with rewiring probability $p$.

\section{Control Simulation}

Because of the strong ability in adaptive learning, neural network is usually used to design intelligent controller for complex industrial system. In addition, neural network can fully approximate any complex nonlinear system. Its identifier can also distinguish the characteristics of uncertain systems with high precision $[28,29]$. In order to establish effective control for linear and nonlinear systems, this paper designs a controller which consists of two kinds of neural network model to obtain good performance. The controller structure is given in Figure 2. As shown in Figure 2, NNI identifies the controlled system online, $e_{1}$ is the error between the reference input and the controlled system output, $e_{2}$ is the error between the identification output and the controlled system output, and $e(t)$ is the error between the system input and the controlled system output. In NNI, the gradient information of $e_{2}$ is used to adjust the weight coefficient.

In Figure 2, NNI is an online identifier of the controlled system with regular structure topology and SNNC is the neural network controller with small-world structure topology. So the principle of small-world neural network control system can be described as follows: NNI identifies the controlled system online, by the use of identification result, then SNNC adjusts the weight coefficients using the identification result and outputs the control variable $u(t)$, and then $u(t)$ is applied to the controlled system and finally make the system output track the setting input to realize adaptive control.

Select the following linear differential system as a controlled system:

$$
\begin{aligned}
y(k)= & 0.33 y(k-1)+0.132 y(k-2) \\
& +0.5 u(k-1)+0.038 u(k-2) .
\end{aligned}
$$

SNNC network structure topology is selected as 3-6-61 (the number of input neurons is 3 , the first and second hidden neurons are 6 , and the output neuron is 1), NNI network structure topology is 3-6-1, the network weights and the thresholds are initialized to the range $[-1,1]$, incremental weight updating strategy is used in Back-Propagation algorithm, and set learning rate $\eta=0.1$, inertia coefficient $\alpha=$ 0.9 , the control period number is 400 , and the error criterion function is defined as follows:

$$
J(k)= \begin{cases}\frac{1}{2}[y(k)-r(k)]^{2}, & \text { SNNC, } \\ \frac{1}{2}[y(k)-\widehat{y}(k)]^{2}, & \text { NNI. }\end{cases}
$$

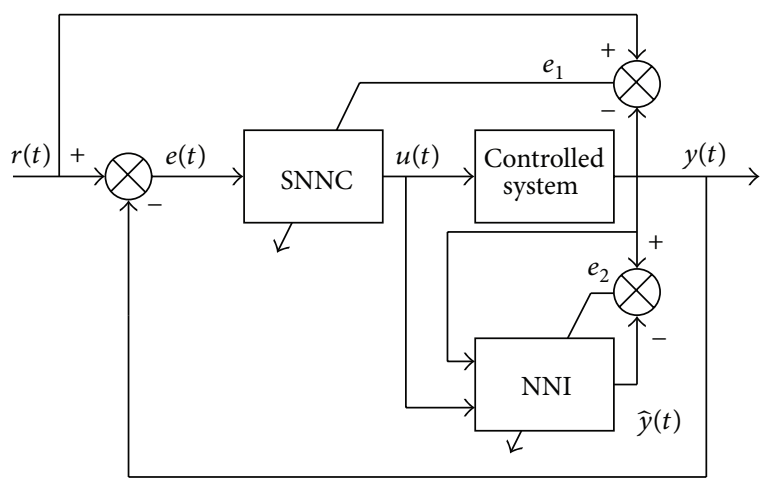

FIGURE 2: Controller with small-world neural network model.

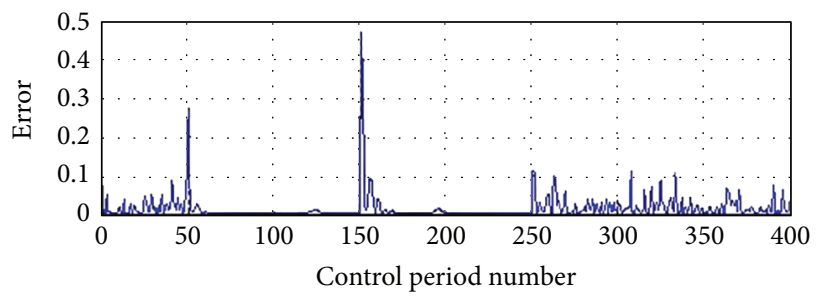

(a) Error curve

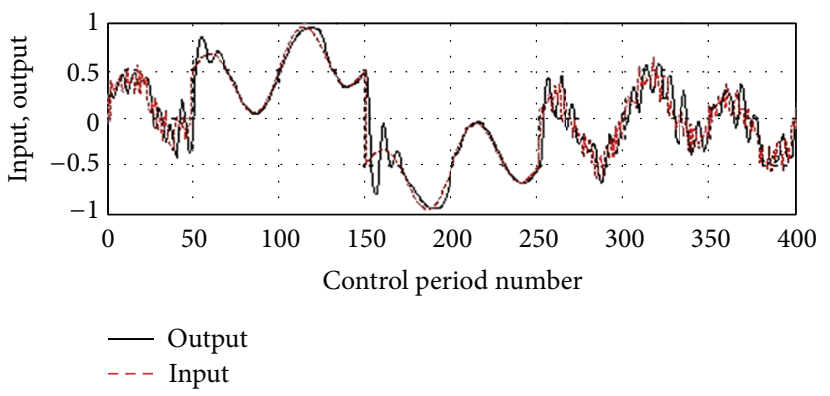

(b) Control curve

FIGURE 3: Linear system $(p=0)$.

Select the input signal $r(k)$ as

$$
\begin{gathered}
r_{1}(k)=0.2 \sin \frac{2 \pi k}{26}+0.3 \sin \frac{\pi k}{15}+0.3 \sin \frac{\pi k}{75}+v_{1}(k), \\
r_{2}(k)=0.3 \sin \frac{2 \pi k}{50}+0.2 \sin \frac{2 \pi k}{100}+v_{2}(k),
\end{gathered}
$$

where,

$$
\begin{gathered}
v_{1}(k)= \begin{cases}0.5, & 50 \leq k \leq 150 \\
-0.5, & 150 \leq k \leq 250 \\
0.1 \times \text { rand }(), & \text { others }\end{cases} \\
v_{2}(k)=0.05 \times \text { rand }() .
\end{gathered}
$$

In the equation, rand() is a random number in the range of $[-1,1]$. In Figure 3 , the input and output curve, the error curve are given when $p=0$ and Figure 4 shows the same curves when $p=0.1$. 


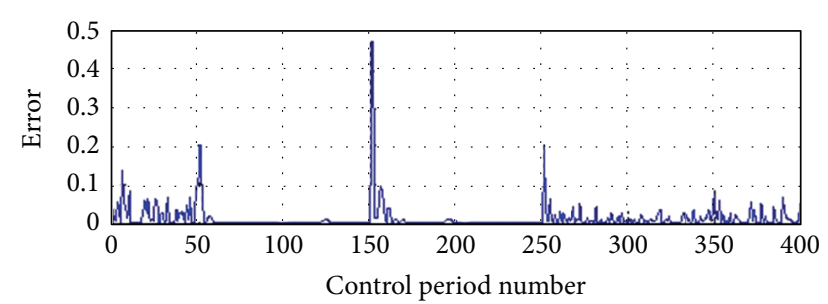

(a) Error curve

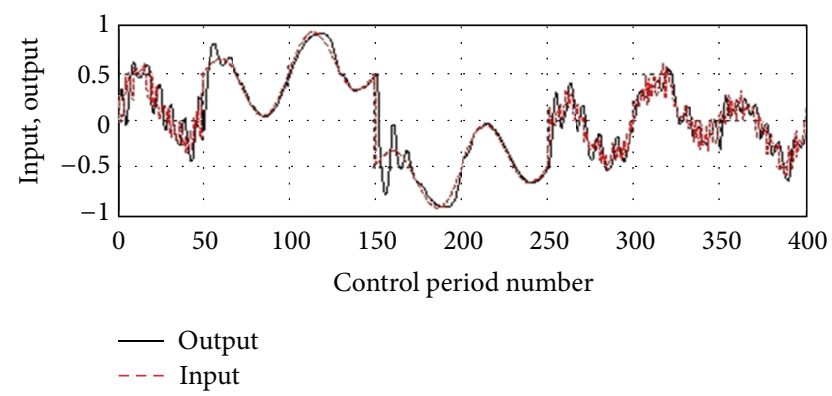

(b) Control curve

Figure 4: Linear system ( $p=0.1)$.

From the figures, we can see that when constant disturbance is added (50 $\leq k \leq 150,150 \leq k \leq 250$ ), neural network control systems all have better ability of selfadapting both $p=0$ and $p=0.1$, regardless of the rewiring probability. The neural network controller can adapt to the impact of constant disturbance through self-learning and adjusting connection weights so quickly that the output of the controlled system catches up with the input signal fast, and it can obtain a smaller error. When adding random disturbance to the input signal, especially within $250 \leq k \leq 400$, the control error of $p=0$ is bigger than that of $p=0.1$ at least by $30 \%$, which means that through rewiring, small-world neural network control system has good anti-interference capability.

Select the following nonlinear system as a controlled system:

$$
y(k)=\frac{1.2 y(k-1)}{\left[2.5+y^{2}(k-1)\right]}+u^{3}(k-1) .
$$

Figures 5 and 6 are given as the input signal and output curve, the error curve when $p=0, p=0.1$. Those diagrams also show that the error is largest when $p=0$. If the input is added with random disturbance, the error is relatively small when $p=0.1$. So small-world neural network control system has a better ability to suppress random disturbances. When the disturbance is a constant, the result is the same for linear system.

\section{Experiment on Electrohydraulic Actuation System}

Presently, the valve-controlled hydraulic servo systems have been applied widely. This kind of system has the features of large output power, fast response, and high accuracy.

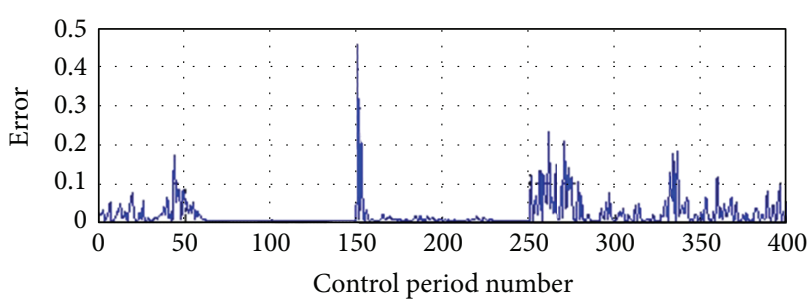

(a) Error curve

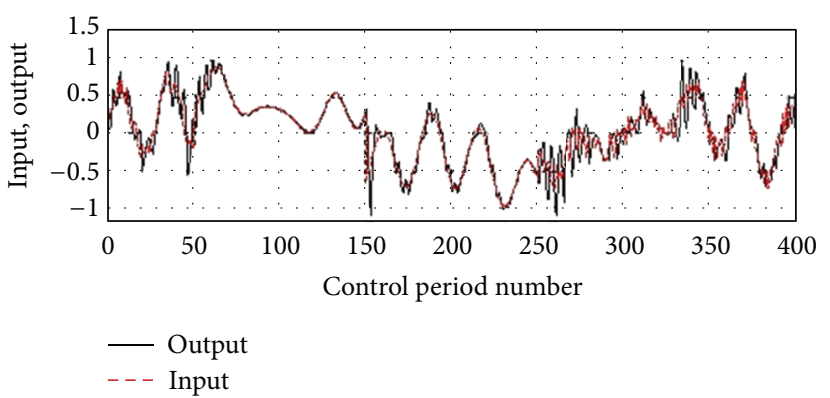

(b) Control curve

FIGURE 5: Nonlinear system $(p=0)$.

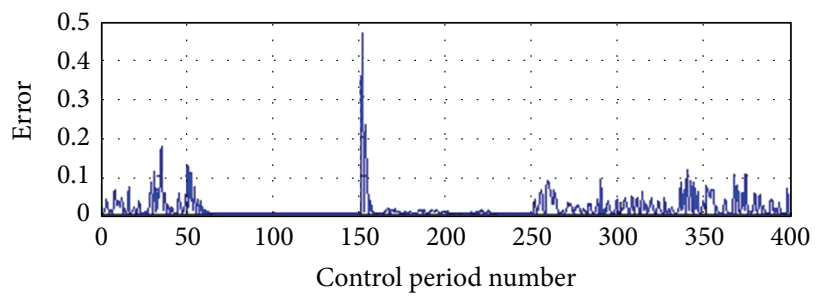

(a) Error curve

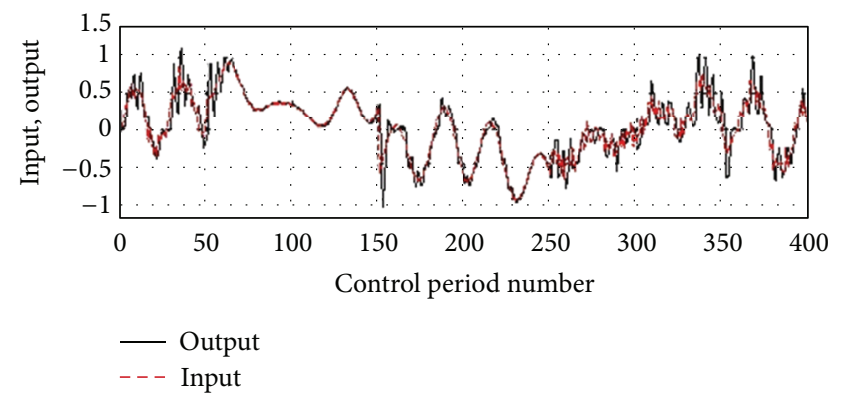

(b) Control curve

Figure 6: Nonlinear system $(p=0.1)$.

However, it also has some shortcomings, such as low reliability, low efficiency, high requirements for oil cleanness, high manufacturing precision for electrohydraulic servo valve, and so on. In order to improve this situation, in recent years, the direct drive electrohydraulic servo system has been widely investigated. A swash plate mechanism is used to control the flow of hydraulic pump in direct drive electrohydraulic servo system. The change of the oblique angle of swash plate, which is regulated by the speed of the direct current motor, may help direct drive variable displacement electrohydraulic position servo control system to change its output flow 


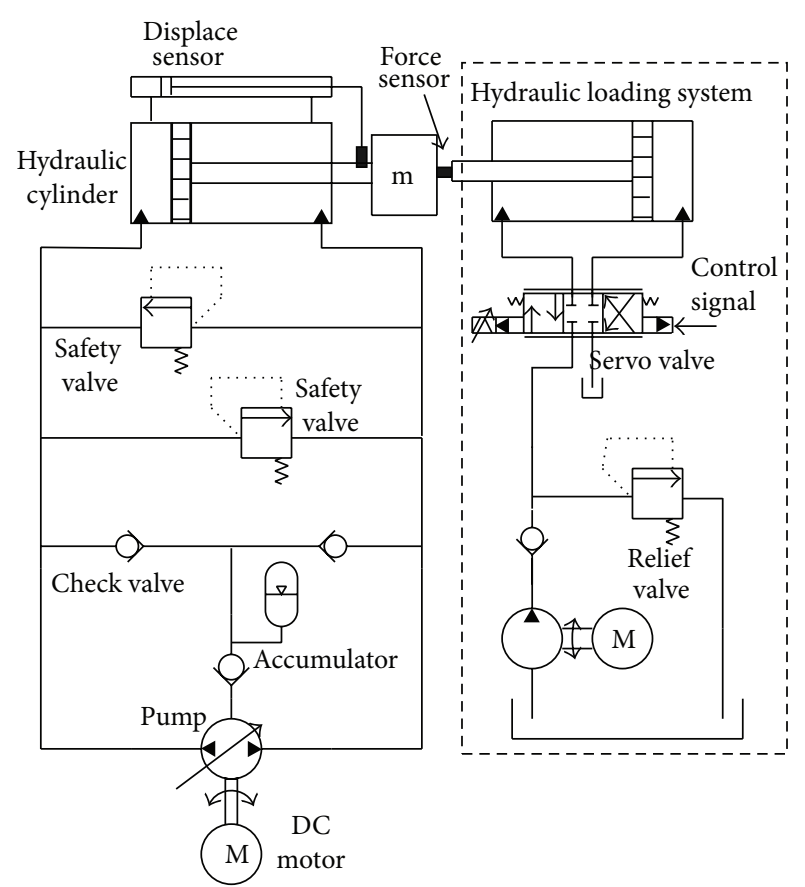

FIGURE 7: Schematic of direct drive variable displacement electrohydraulic actuation position servo control system (including hydraulic loading system).

and consequently serve to control the position of hydraulic actuator. Because of the high efficiency, easy operation, and big output force of this kind of system, it has been applied in many industry fields, such as electrohydraulic actuation system, precision forging machines, marine steering gear, and injection molding machines [30-34]. Due to existing nonlinear factors, it is difficult to establish the accurate mathematical model of this system. Figure 7 shows the schematic of direct drive variable displacement electrohydraulic actuation position control system. As shown in Figure 7, the hydraulic loading system is used to simulate the external load, and the control platform can control the hydraulic loading system to output different force value. The control objective of this electrohydraulic actuation system is to accurately track the given position signal, in which tracking performance, fast response, high accuracy, and good anti-interference must be taken into account. Therefore, the control strategy of this system is very important, and the small-world neural network controller is adopted to control this system given that it does not need accurate mathematical model.

Hardware configuration of electrohydraulic actuation system is listed in Table 1.

Firstly, in the case of no external load applied on direct drive variable displacement electrohydraulic actuation position system, the system is controlled to track square wave signal using small-world neural network. Figure 8 shows the tracking result of $1 \mathrm{~Hz}$ square wave signal with peak value of $200 \mathrm{~mm}$, where the curve of input signal is marked A, and output is marked B. The following diagrams are similar. As shown in the figure, after 2 cycles of learning, the system output signal can track the target displacement, the response
TABLE 1: Hardware list of electro-hydraulic actuation system.

\begin{tabular}{lcl}
\hline Name & Type & Specification \\
\hline $\begin{array}{l}\text { Brushless DC } \\
\text { motor }\end{array}$ & BLF & Three phases $300 \mathrm{~W}$ \\
\hline $\begin{array}{l}\text { Displacement } \\
\text { sensor }\end{array}$ & LVDT & $\pm 400 \mathrm{~mm}$ \\
\hline $\begin{array}{l}\text { Angular } \\
\text { displacement } \\
\text { sensor }\end{array}$ & RVDT & $\pm 30^{\circ}$ \\
\hline $\begin{array}{l}\text { Swash plate } \\
\text { hydraulic pump }\end{array}$ & $\begin{array}{c}\text { Displacement } \\
\text { pump }\end{array}$ & $\begin{array}{l}\text { Swash plate angle: } \pm 20^{\circ} \\
\text { Maximum displacement: } \\
84 \text { mL } / \mathrm{r}\end{array}$ \\
\hline $\begin{array}{l}\text { Hydraulic cylinder } \\
\text { Single-acting } \\
\text { hydraulic cylinder }\end{array}$ & $\begin{array}{l}\text { Stroke: } 800 \mathrm{~mm} \\
\text { Internal diameter: } 50 \mathrm{~mm}\end{array}$ \\
\hline
\end{tabular}

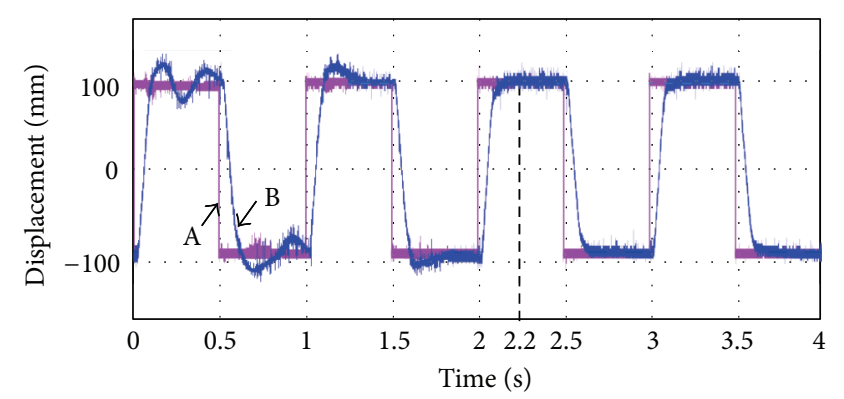

Figure 8: Track control of $1 \mathrm{~Hz}$ square wave (no load).

time is about $0.2 \mathrm{~s}$, and the steady state error is less than $0.2 \mathrm{~mm}$.

This result shows that the use of small-world neural network control strategy for position control obtains satisfactory control precision and response speed in the case of noload. The structure topology of small-world neural network used in control test is the same as the simulation test.

Figure 9 shows the track control result by the use of small-world neural network control strategy under $10000 \mathrm{~N}$ external load. The hydraulic cylinder is controlled to track $2 \mathrm{~Hz}$, peak value of $200 \mathrm{~mm}$ square wave signal. The result shows that the system has rapid response, about $0.25 \mathrm{~s}$. The steady-state error is slightly larger, at about $2 \mathrm{~mm}$. It indicates that the influence of external load will be further weakened by small-world neural network controller. Since the electrohydraulic actuation control system can also achieve fast tracking square wave signal, it can obtain satisfactory control accuracy under $10000 \mathrm{~N}$ external load.

The load under actual situation usually appears quickly and unexpectedly. In order to testify whether the small-world neural network control strategy performs well or not, we simulate the step response control in the case of impact load 5000 N. The result is shown in Figure 10.

As shown in the figure, at $3.7 \mathrm{~s}$, the impact load $5000 \mathrm{~N}$ is added, and after about $0.25 \mathrm{~s}$, the electrohydraulic actuation system can correct the error caused by the change of external load. The steady state error can be controlled within $0.2 \mathrm{~mm}$ or less. It shows that the small-world neural network 


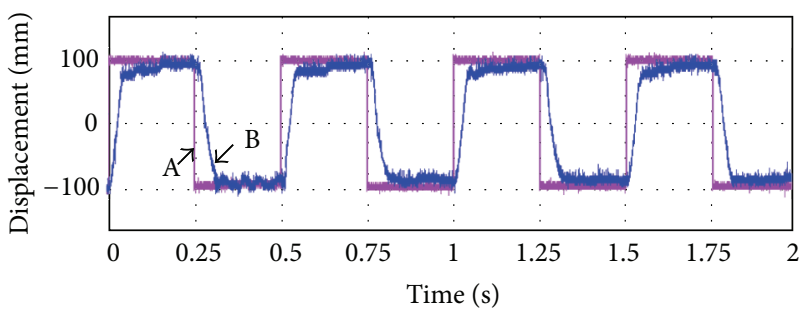

FIGURE 9: Track control of $2 \mathrm{~Hz}$ square wave (10000 N load).

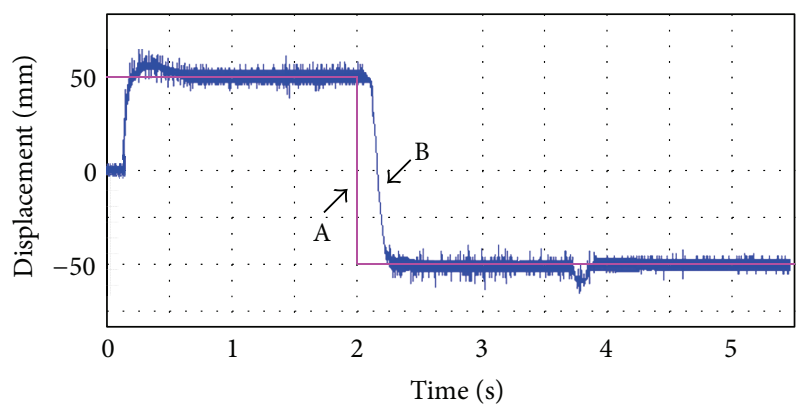

FIGURE 10: Step control curve (impact load 5000 N).

controller has a better ability to suppress the impact of interference. The digital simulation results are basically consistent with the experimental results under external interference.

\section{Conclusions}

In this paper, a multilayer feed forward neural network model is proposed through reconnecting the regular links which relies heavily on the rewiring probability, and then a controller based on small-world neural network model is designed to control linear and nonlinear systems. Simulation results show that the regular and the small-world network all have better control performance under constant disturbance. But when adding random disturbance, the small-world neural network control system is superior to the corresponding regular neural network control system in control accuracy. So the multilayer small-world neural network control system has good anti-interference features. Furthermore, no matter there is load or not, the small-world neural network controller for direct drive electrohydraulic actuation position control system can obtain faster response, better control precision, and an ability of anti-interference, which means that small-world neural network can be used to develop intelligent controller for industrial systems. However, it still needs further study on how to obtain the optimal rewiring probability to make the best of the controller's performance.

\section{Acknowledgment}

This work is supported in part by the National Natural Science Foundation of China under Grant no. 51105297.

\section{References}

[1] D. Batalle, E. Eixarch, F. Figueras et al., "Altered smallworld topology of structural brain networks in infants with intrauterine growth restriction and its association with later neurodevelopmental outcome," NeuroImage, vol. 60, no. 2, pp. 1352-1366, 2012.

[2] M. Bolaños, E. M. Bernat, B. He, and S. Aviyente, "A weighted small world network measure for assessing functional connectivity," Journal of Neuroscience Methods, vol. 212, no. 1, pp. 133142, 2013.

[3] O. Sporns and C. J. Honey, "Small worlds inside big brains," Proceedings of the National Academy of Sciences of the United States of America, vol. 103, no. 51, pp. 19219-19220, 2006.

[4] R. Albert and A. L. Barabási, "Statistical mechanics of complex networks," Reviews of Modern Physics, vol. 74, no. 1, pp. 47-97, 2002.

[5] J. Lu, D. W. C. Ho, and Z. Wang, "Pinning stabilization of linearly coupled stochastic neural networks via minimum number of controllers," IEEE Transactions on Neural Networks, vol. 20, no. 10, pp. 1617-1629, 2009.

[6] J. Cao, P. Li, and W. Wang, "Global synchronization in arrays of delayed neural networks with constant and delayed coupling," Physics Letters A, vol. 353, no. 4, pp. 318-325, 2006.

[7] Y. Xia and J. Wang, "A general methodology for designing globally convergent optimization neural networks," IEEE Transactions on Neural Networks, vol. 9, no. 6, pp. 1331-1343, 1998.

[8] S. Hu, X. Liao, and X. Mao, "Stochastic Hopfield neural networks," Journal of Physics A, vol. 36, no. 9, pp. 2235-2249, 2003.

[9] K. O. Stanley, D. B. D’Ambrosio, and J. Gauci, "A hypercubebased encoding for evolving large-scale neural networks," Artificial Life, vol. 15, no. 2, pp. 185-212, 2009.

[10] O. R. de Lautour and P. Omenzetter, "Prediction of seismicinduced structural damage using artificial neural networks," Engineering Structures, vol. 31, no. 2, pp. 600-606, 2009.

[11] E. Gelenbe and J. M. Fourneau, "Random neural networks with multiple classes of signals," Neural Computation, vol. 11, no. 4, pp. 953-963, 1999.

[12] D. J. Watts and S. H. Strogatz, "Collective dynamics of 'smallworld9 networks," Nature, vol. 393, no. 6684, pp. 440-442, 1998.

[13] G. A. Pagani and M. Aiello, "The power grid as a complex network: a survey," Physica A, vol. 392, no. 11, pp. 2688-2700, 2013.

[14] F. Gerhard, G. Pipa, B. Lima, S. Neuenschwander, and W. Gerstner, "Extraction of network topology from multi-electrode recordings: is there a small-world effect?" Frontiers in Computational Neuroscience, vol. 5, no. 4, pp. 1-13, 2011.

[15] H. D. Rozenfeld, C. Song, and H. A. Makse, "Small-world to fractal transition in complex networks: a renormalization group approach," Physical Review Letters, vol. 104, no. 2, Article ID 025701, 2010.

[16] A. R. Backes, D. Casanova, and O. M. Bruno, "A complex network-based approach for boundary shape analysis," Pattern Recognition, vol. 42, no. 1, pp. 54-67, 2009.

[17] M. Karsai, M. Kivelä, R. K. Pan et al., "Small but slow world: how network topology and burstiness slow down spreading," Physical Review E, vol. 83, no. 2, Article ID 025102, 2011.

[18] C. Wu and B. Zhou, "Complex network analysis of tag as a social network," Journal of Zhejiang University, vol. 44, no. 11, pp. 21942197, 2010 (Chinese). 
[19] V. M. Eguíluz, D. R. Chialvo, G. A. Cecchi, M. Baliki, and A. V. Apkarian, "Scale-free brain functional networks," Physical Review Letters, vol. 94, no. 1, Article ID 018102, 2005.

[20] D. S. Bassett and E. Bullmore, "Small-world brain networks," Neuroscientist, vol. 12, no. 6, pp. 512-523, 2006.

[21] J. Piersa, F. Piekniewski, and T. Schreiber, "Theoretical model for mesoscopic-level scale-free self-organization of functional brain networks," IEEE Transactions on Neural Networks, vol. 21, no. 11, pp. 1747-1758, 2010.

[22] C. Li, "Memorizing morph patterns in small-world neuronal network," Physica A, vol. 388, no. 2-3, pp. 240-246, 2009.

[23] S. Lu, J. Fang, A. Guo, and Y. Peng, "Impact of network topology on decision-making," Neural Networks, vol. 22, no. 1, pp. 30-40, 2009.

[24] O. Erkaymaz, M. Özer, and N. Yumuşakc, "Performance analysis of a feed forward artifical neural network with small-world topology," Procedia Technology, vol. 1, pp. 291-296, 2012.

[25] D. Simard, L. Nadeau, and H. Kröger, "Fastest learning in smallworld neural networks," Physics Letters A, vol. 336, no. 1, pp. 815, 2005.

[26] G. Trtnik, F. Kavčič, and G. Turk, "Prediction of concrete strength using ultrasonic pulse velocity and artificial neural networks," Ultrasonics, vol. 49, no. 1, pp. 53-60, 2009.

[27] V. Kůrková, P. C. Kainen, and V. Kreinovich, "Estimates of the number of hidden units and variation with respect to halfspaces," Neural Networks, vol. 10, no. 6, pp. 1061-1068, 1997.

[28] Z. Z. Yuan, Z. Q. Chen, and X. Li, "Connectionism intelligent control: a survey," Acta Automatica Sinica, vol. 28, no. 1, pp. 3859, 2002 (Chinese).

[29] H. M. R. Ugalde, J. C. Carmona, V. M. Alvarado, and J. ReyesReyes, "Neural network design and model reduction approach for black box nonlinear system identification with reduced number of parameters," Neurocomputing, vol. 101, pp. 170-180, 2013.

[30] S. Habibi, R. Burton, and E. Sampson, "High precision hydrostatic actuation systems for micro- and nanomanipulation of heavy loads," Transactions of the ASME, vol. 128, no. 4, pp. 778787, 2006.

[31] S. R. Habibi and R. Burton, "Parameter identification for a highperformance hydrostatic actuation system using the variable structure filter concept," Transactions of the ASME, vol. 129, no. 2, pp. 229-235, 2007.

[32] J. L. Liu, J. H. Jiang, T. R. Lu, Q. H. Liu, C. W. Zhang, and H. G. $\mathrm{Xu}$, "Direct drive volume control electro-hydraulic servo active mass driver system," Journal of Harbin Institute of Technology, vol. 43, no. 9, pp. 51-55, 2011 (Chinese).

[33] K. M. Elbayomy, Z. Jiao, and H. Zhang, "PID controller optimization by GA and its performances on the electro-hydraulic servo control system," Chinese Journal of Aeronautics, vol. 21, no. 4, pp. 378-384, 2008.

[34] J. Yao, Z. Jiao, Y. Shang, and C. Huang, "Adaptive nonlinear optimal compensation control for electro-hydraulic load simulator," Chinese Journal of Aeronautics, vol. 23, no. 6, pp. 720-733, 2010. 


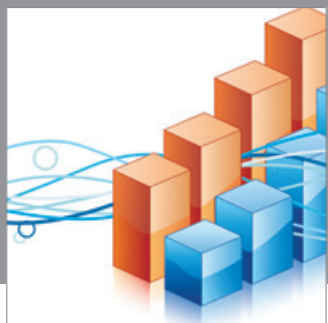

Advances in

Operations Research

mansans

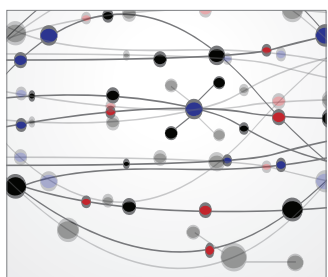

The Scientific World Journal
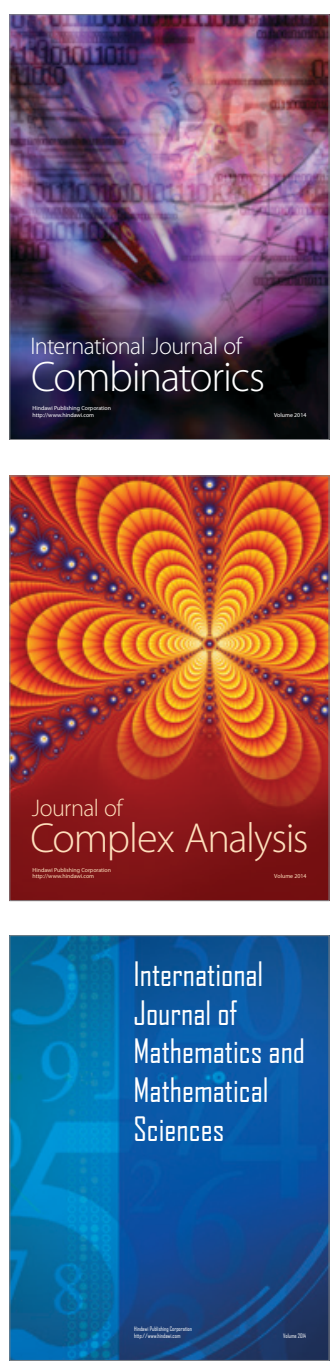
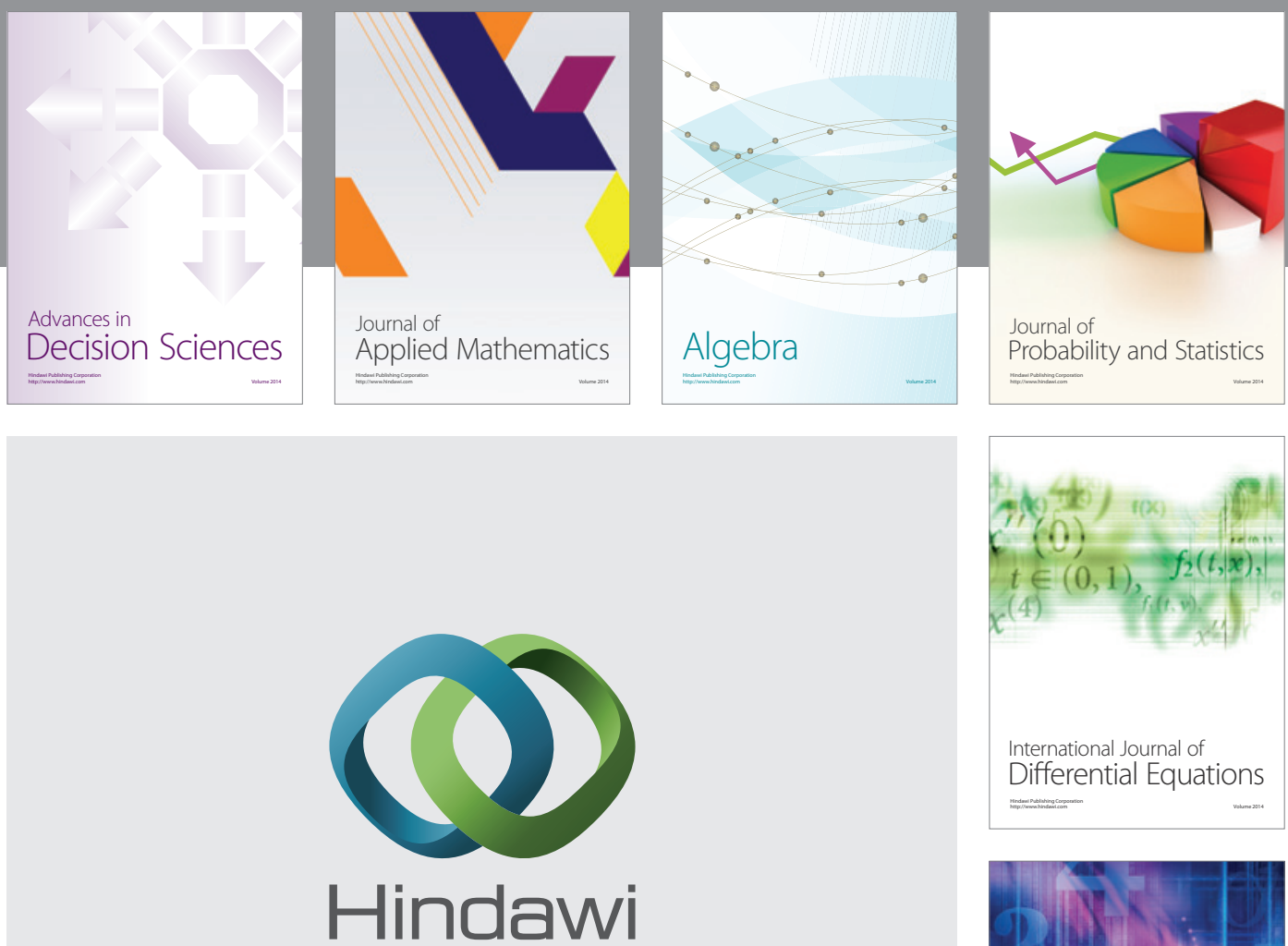

Submit your manuscripts at http://www.hindawi.com
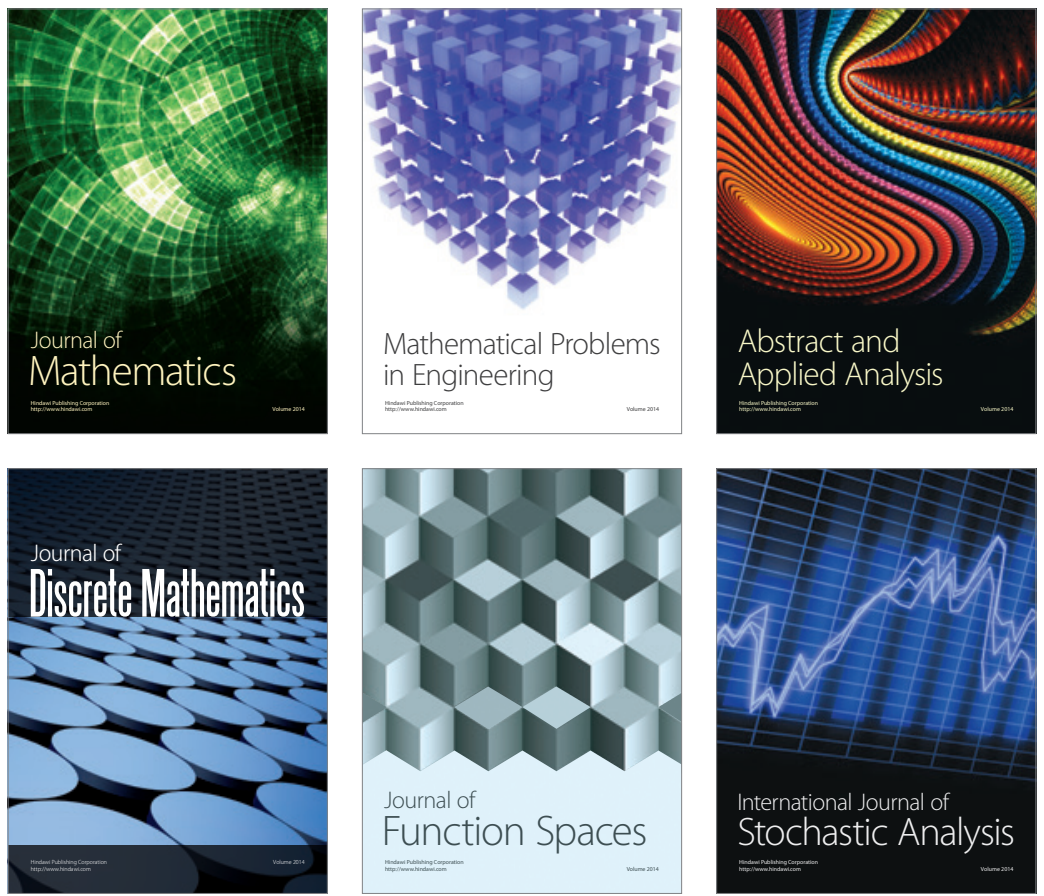

Journal of

Function Spaces

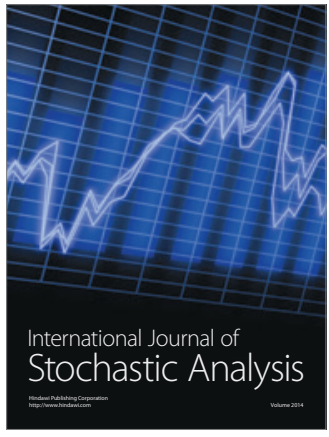

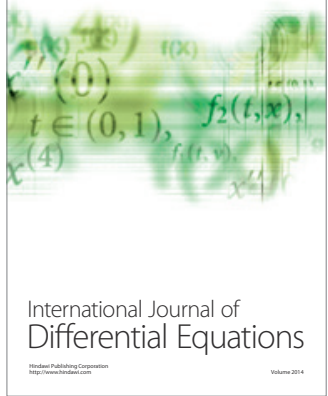
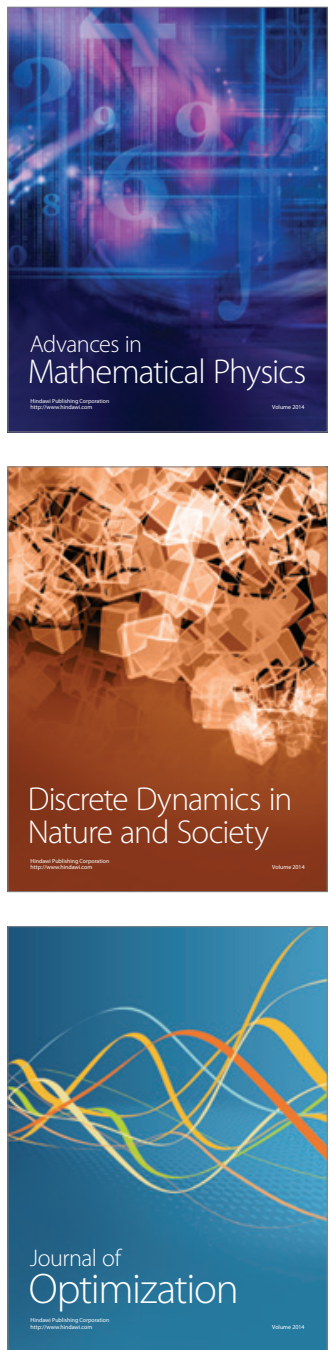